Draft version March 27, 2022

Preprint typeset using $\mathrm{LAT}_{\mathrm{E}} \mathrm{X}$ style emulateapj v. 5/2/11

\title{
CONSTRAINING THE CMB OPTICAL DEPTH THROUGH THE DISPERSION MEASURE OF COSMOLOGICAL RADIO TRANSIENTS
}

\author{
Anastasia Fialkov \& Abraham Loeb \\ Institute for Theory and Computation, Harvard University, 60 Garden Street, $M S-51$, Cambridge, MA, 02138 U.S.A. \\ Draft version March 27, 2022
}

\begin{abstract}
The dispersion measure of extragalactic radio transients, such as of recently discovered Fast Radio Burst FRB150418, can be used to measure the column density of free electrons in the intergalactic medium. The same electrons also scatter the Cosmic Microwave Background (CMB) photons, affecting precision measurements of cosmological parameters. We explore the connection between the dispersion measure of radio transients existing during the Epoch of Reionization (EoR) and the total optical depth for the CMB, $\tau_{C M B}$, showing that the existence of such transients would provide a new sensitive probe of $\tau_{C M B}$. As an example, we consider the population of FRBs. Assuming they exist during the EoR, we show that: (i) such sources can probe the reionization history by measuring $\tau_{C M B}$ to sub-percent accuracy, and (ii) they can be detected with high significance by an instrument such as the Square Kilometer Array.

Subject headings: cosmology: cosmological parameters
\end{abstract}

\section{INTRODUCTION}

The Epoch of Reionization (EoR) is one of the major research areas in cosmology today (Loeb \& Furlanetto 2013). It started around $z \sim 13$, and by redshift of $z \sim 6$ most of the hydrogen atoms in the intergalactic medium were ionized by the UV photons emitted by stars and quasars (Zahn et al. 2012; George 2015; Ade et al. 2015; Becker et al. 2015). Hydrogen reionization was closely followed by first helium reionization during which helium atoms lost their outer electron, while second helium reionization occurred around $z \sim 3$. The reionization of the intergalactic medium (IGM) is a nuisance for the Cosmic Microwave Background (CMB) cosmology as CMB photons scatter off the ionized gas. The precision with which the total optical depth to reionization, $\tau_{C M B}$, can be measured from the CMB data is very poor, e.g., the $68 \%$ confidence level in $\tau_{C M B}$ corresponds to a relative error of $\sim 24 \%$ (Ade et al. 2015). Because so little is known about the EoR at present, the large uncertainty in $\tau_{C M B}$ propagates to other cosmological parameters extracted from the CMB data. Luckily, alternative probes of reionization, such as the 21-cm line of hydrogen, will in the future provide independent constraints on $\tau_{C M B}$ and help to remove the related uncertainty (Liu et al. 2015; Fialkov \& Loeb 2016).

In addition to the CMB scattering, the ionized plasma leaves other fingerprints on observed radiation. Any radio waves will follow the dispersion relation $w^{2}=$ $w_{p}^{2}+k^{2} c^{2}$ as they propagate through cold unmagnetized plasma with electron density $n_{e}$, where $w=2 \pi \nu$ with $\nu$ being the frequency of the wave and $k$ its wavenumber, $c$ is the speed of light, and $w_{p}=5.641 \times 10^{4}\left(n_{e} / \mathrm{cm}^{-3}\right)^{1 / 2}$ $\mathrm{s}^{-1}$ is the plasma frequency. As a result, the group velocity of any radio wave packet is modified in a frequencydependent way as the signal travels through the ionized plasma. In such a medium, a transient at frequency $\nu$

anastasia.fialkov@cfa.harvard.edu

aloeb@cfa.harvard.edu is delayed by $\Delta t=4.15 \times 10^{-3} \mathrm{DM} / \nu^{2}$ seconds, where $\nu$ is in $\mathrm{GHz}$ and the dispersion measure, $\mathrm{DM}$, is defined as the integrated electron density along the line of sight in units of $\mathrm{pc} \mathrm{cm}^{-3}$. For extragalactic sources, the DM includes the contributions from the interstellar medium of the Milky Way, the host galaxy of the source, and the IGM. For sources at high redshifts $(z \gtrsim 0.5)$, the ionized IGM should be the dominant source of dispersion (DM $\gtrsim 500 \mathrm{pc} \mathrm{cm}^{-3}$ ) without substantial contamination from the Milky Way, the local Universe and the host galaxy $\left(\mathrm{DM} \lesssim 250 \mathrm{pc} \mathrm{cm}^{-3}\right)$. Thus, measuring the dispersion of high-redshift sources offers a unique way to probe the ionized IGM and constrain the cosmic reionization history. In particular, the dispersion of radio emission by high redshift Gamma Ray Bursts (GRB) was discussed as a probe of the IGM by Ginzburg (1973), Palmer (1993), Ioka (2003) and Inoue (2004), and the recently discovered Fast Radio Burst (FRB) FRB150418, associated with a galaxy at $z \sim 0.492$, provided a direct measurement of the cosmic baryon density of the IGM of 4.9\% through its DM measurement (Keane et al. 2016).

Although both the dispersion measure and $\tau_{C M B}$ probe the same column density of the free electrons, the connection between these two quantities has not been made in the literature. Assuming that radio transients exist at high enough redshifts, we discuss their cosmological implications in Section 2 focusing on the relation between $\tau_{C M B}$ and DM and showing that an ensemble of high-redshift radio transients would probe $\tau_{C M B}$ to subpercent accuracy. In Section 3 we discuss perspectives for such measurement. Assuming that radio transients, such as the population of FRBs, exist at high enough redshifts, we show that the Square Kilometer Array (SKA) will have sufficient sensitivity to probe these sources out to $z \sim 14$ and fully constrain the history of reionization through their DM. We conclude in Section 4. Throughout this work we use cosmological parameters $H_{0}, Y_{P}$, $\Omega_{b}, \Omega_{m}$ and $\Omega_{\Lambda}$ from Ade et al. (2015).

${ }^{1}$ https://www.skatelescope.org/ 


\section{2. $\tau$-DM RELATION}

We start by exploring the connection between the dispersion measure in the signal of a cosmological transient at a redshift $z$

$$
\operatorname{DM}(z)=\int_{0}^{z} \frac{n_{e}\left(z^{\prime}\right)}{1+z^{\prime}} d l
$$

and the contribution of the IGM at redshifts out to $z$ to the total optical depth of the CMB,

$$
\tau(z)=\int_{0}^{z} \sigma_{T} n_{e}\left(z^{\prime}\right) d l
$$

where $\sigma_{T}=6.25 \times 10^{-25} \mathrm{~cm}^{2}$ is the Thompson cross-section, $d l=c d t=-c d z^{\prime} /\left(1+z^{\prime}\right) H\left(z^{\prime}\right)$ is the differential of the proper distance and $H(z)=$ $H_{0}\left[\Omega_{m}(1+z)^{3}+\Omega_{\Lambda}\right]^{1 / 2}$ is the Hubble parameter. Taking $z$ to the beginning of the EoR provides the total optical depth of the CMB, $\tau_{C M B}$. Both DM and $\tau(z)$ depend on the redshift evolution of electron density

$$
n_{e}(z)=\bar{n}_{b}(z)\left[\left(1-Y_{P}\right) x_{H I I}+\frac{Y_{P}}{4}\left(x_{H e I I}+x_{H e I I I}\right)\right]
$$

where $\bar{n}_{b}(z)=\Omega_{b} \rho_{c r, 0}(1+z)^{3} / m_{p}$ is the mean baryon density at redshift $z, \rho_{c r, 0}$ is the critical density today, $m_{p}$ is the proton mass, and $Y_{P}$ is the helium mass fraction. The redshift evolution of the electron density depends on the details of reionization. In the above equation $x_{H I I}$ is the redshift-dependent hydrogen ionization fraction, $x_{H e I I}$ is the fraction of singly ionized helium and $x_{H e I I I}$ is the fraction of fully ionized helium.

In the left panel of Figure 1 we show examples of $n_{e}(z)$ for three different models of the reionization history each shown for two values of $\tau_{C M B}=0.057$ and 0.078 , both within $1-\sigma$ confidence level of the recent Planck satellite measurement $\tau_{C M B}=0.066 \pm 0.016$ (Ade et al. 2015). Assuming that hydrogen and first helium reionization occur simultaneously due to their similar ionization potentials (i.e., $x_{H I I}=x_{H e I I}$ ), we adopt the following models (i) an instantaneous reionization where the hydrogen ionization fraction jumps from 0 to 1 at $z_{r e}=8.1$ (10.7) for $\tau_{C M B}=0.057(0.078)$, (ii) a reionization history parameterized by the conventional form

$$
x_{H I I}=\frac{1}{2}\left[1-\tanh \left(\frac{z-z_{r e}}{\Delta z_{r e}}\right)\right]
$$

for the same values of $z_{r e}$ and $\Delta z_{r e}=1$, and (iii) a realistic reionization history which for $\tau_{C M B}=0.078$ assumes that star formation occurs in halos with mass larger than the atomic cooling threshold, $\mathrm{M}_{\mathrm{h}} \gtrsim 10^{8}[(1+z) / 10]^{-3 / 2}$ $\mathrm{M}_{\odot}$, and accounts for the effect of the photoheating feedback on star formation (Cohen et al. 2015), while in the case of $\tau_{C M B}=0.057$ only very massive halos contributed to reionization, $\mathrm{M}_{\mathrm{h}} \gtrsim 10^{9}[(1+z) / 10]^{-3 / 2} \mathrm{M}_{\odot}$. Finally, we assume a simple model for second helium reionization with $\mathrm{HeIII}$ fraction being of the same form as in Eq. (4) with $z_{r e}=3$ and $\Delta z_{r e}=1$.

Figure 1] also shows the integrated optical depth out to redshift $z$ (middle panel) and the DM of a source at redshift $z$ (right panel) for the three reionization histories and the two values of $\tau_{C M B}$. Both DM and $\tau$ saturate at high redshifts before the beginning of reionization when the free electron fraction drops to zero. Depending on the normalization, the value to which $\tau(z)$ saturates is either $\tau_{C M B}=0.078$ or $\tau_{C M B}=0.057$, while the upper limit on the DM is slightly different for each reionization history. For the high optical depth the DM takes the values of $7225 \mathrm{pc} \mathrm{cm}^{-3}$ in the case of instanteneous EoR, 7213 pc $\mathrm{cm}^{-3}$ for the tanh model and $7101 \mathrm{pc} \mathrm{cm}^{-3}$ for the more realistic scenario; while for the low optical depth the values are 6215,6190 and $6137 \mathrm{pc} \mathrm{cm}^{-3}$ respectively.

Although the dependence of the dispersion measure of high-redshift transients on the reionization history has been explored in literature (Ioka 2003; Inoue 2004), the connection between the intergalactic DM and $\tau(z)$ has not been made. Given a series of the DM measurements out to redshift $z$ for an extragalactic transient population, $\tau(z)$ can be computed precisely. Using integration by parts we can analytically derive the relation between $\tau(z)$ and $\operatorname{DM}(z)$

$$
\tau(z)=\left[\frac{\mathrm{DM}(z)}{\mathrm{cm}^{-2}}(1+z)-\int_{0}^{z} \frac{\mathrm{DM}\left(z^{\prime}\right)}{\mathrm{cm}^{-2}} d z^{\prime}\right] \times \sigma_{T} .
$$

An example for $\tau$ as a function of the DM is shown in Figure 2, Probing the evolution of the DM with redshifts gives a measure of $\tau(z)$ and integrating over the whole range of the DM up to its maximal value should allow one to derive $\tau_{C M B}$. The shape of the $\tau(\mathrm{DM})$ curve has a weak dependence on the reionization history. For the same tanh model of EoR, changing the duration of reionization from $\Delta z_{r e}=1$ to $\Delta z_{r e}=4$ decreases the value of the DM by $\sim 1.4 \%$ which is of order the contribution from the interstellar medium inside the Milky Way to the total DM and is small compared to the extragalactic contribution for cosmological transients.

The error in the determination of the optical depth can be expressed as

$$
\Delta \tau(z)=\left[\frac{\Delta \mathrm{DM}(z)}{\mathrm{cm}^{-2}}(1+z)-\int_{0}^{z} \frac{\Delta \mathrm{DM}\left(z^{\prime}\right)}{\mathrm{cm}^{-2}} d z^{\prime}\right] \times \sigma_{T}
$$

where to leading order the uncertainty due to the redshift error $\Delta z$ cancels out. If cosmological transients are detected during the EoR this method is expected to provide $\tau_{C M B}$ measurements to a good precision. Assuming a constant error of $\triangle \mathrm{DM}=100 \mathrm{pc} \mathrm{cm}{ }^{-3}$ (typical uncertainty due to host galaxies) we get $\Delta \tau / \tau \sim 0.3 \%$.

\section{OBSERVATIONAL PROSPECTS}

There is no doubt that probing the reionization history with highly dispersed transients is very challenging, requiring: (i) bright radio transients at high enough redshifts, and (ii) sensitive enough telescope which is designed to probe the corresponding time delays. Here we consider a possibility that recently discovered FRBs could play the role of cosmological beacons and regard the future SKA as a tool to probe them.

\subsection{FRBs as Cosmological Beacons}

FRBs are short $(\sim$ millisecond $)$, bright $(\sim \mathrm{Jy})$ pulses at radio frequencies $(\sim 1 \mathrm{GHz})$ observed over the entire sky (Katz 2016; Petroff et al. 2016; Keane et al. 2016). Out of 17 known FRBs (Petroff et al. 2016; Keane et al. 2016) 15 bursts, including the first FRB discovered in 2007 (Lorimer et al. 

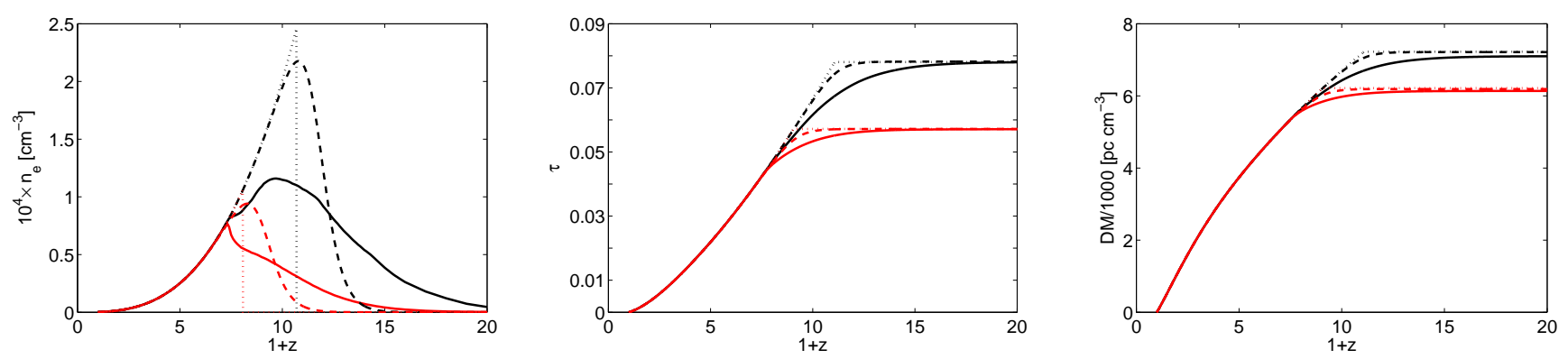

FIG. 1. - Rescaled free electron density, $10^{4} \times n_{e}(z)\left[\mathrm{cm}^{-3}\right]$, at redshift $z$ (left), integrated CMB optical depth out to redshift $z$ (middle panel) and rescaled dispersion measure $\left(\mathrm{DM} / 1000\left[\mathrm{pc} \mathrm{cm}^{-3}\right]\right)$ for a source at redshift $z$ (right) are shown for a realistic reionization scenarion (solid), tanh model of reionization (dashed) and an instanteneous reionization (dotted) for $\tau=0.057$ (red) and $\tau=0.078$ (black).

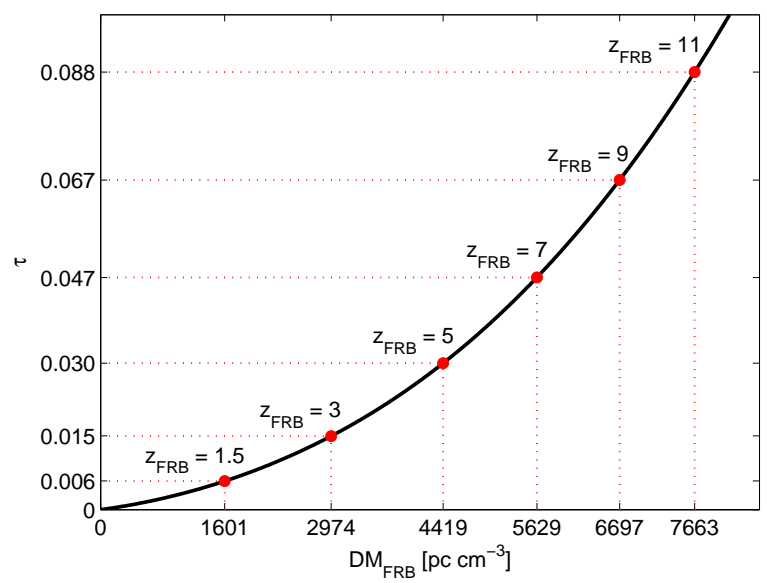

FIG. 2.- $\tau$ as a function of the DM in a fully ionized IGM. For a transient at redshift $z_{F R B}$ the horizontal and vertical dotted lines mark $\tau\left(z_{F R B}\right)$ and the DM of the transient respectively.

2007), were found in the data collected by Parkes Radio Telescope in the radio band with central frequency of $\sim 1372.5 \mathrm{MHz}$ and bandwidth of $288 \mathrm{MHz}$ (Lorimer et al. 2007; Keane et al. 2011; Thornton et al. 2013; Burke-Spolaor \& Bannister 2014; Champion et al. 2015; Petroff et al. 2015; Ravi et al. 2015; Keane et al. 2016), one FRB was detected in the Arecibo Pulsar ALFA Survey at 1225-1525 $\mathrm{MHz}$ frequencies (Spitler et al. 2014) and one FRB was found in the Green Bank Telescope (GBT) data within the 700-900 MHz frequency range (Masui et al. 2015). These bursts are very bright pulses with the peak detected flux, $S_{\text {peak }}$, being in the 0.3-1.3 Jy range for all the bursts except for the original "Lorimer" burst which had $S_{\text {peak }}>30$ Jy (Lorimer et al. 2007). Except for recently discovered FRB150418 which was followed by a $\sim 6$ day radio afterglow, the radio bursts observed so far are not known to be accompanied by emission in other wavelength regimes, such as X-rays or gamma-rays, making their identification more difficult.

The short duration of FRBs allows one to probe the delay time at each frequency. As for pulsars, the observed delay scales as $\nu^{-2}$ (Keane et al. 2011; Thornton et al. 2013; Burke-Spolaor \& Bannister 2014; Masui et al. 2015; Petroff et al. 2015), which can be explained by dispersion in a cold plasma. However, the typical DM for FRBs, DM $=375-1629 \mathrm{pc} \mathrm{cm}^{-3}$, is much larger than what is usually measured for the Galactic transients, $\mathrm{DM} \lesssim 250 \mathrm{pc} \mathrm{cm}^{-3}$. Therefore, an extragalactic expla- nation for the observed FRBs, which was recently supported by the host galaxy identification for FRB150418 (Keane et al. 2016), is usually invoked (Lvubarsky 2014; Totani 2013; Zhang 2014; Geng \& Huang 2015; Falcke \& Rezzolla 2014) with the observed DM of FRBs corresponding to their redshifts in the range $z \sim 0.2-1.4$. However, there might be several distinct populations of sources contributing to the discovered FRBs, and there are theoretical models where the observed high DM of FRBs is be produced locally (Kulkarni et al. 2014; Loeb et al. 2014).

In the following we consider a population of FRBs similar to the detected ones but located at much higher redshifts during the EoR and consider prospects for their detection. Using the existing data we first estimate the redshift of each known $\mathrm{FRB}, z_{0}$, relying on the observed dispersion measure (Petroff et al. 2015). For our purposes, the DM contributions from both the Milky Way and the host FRB galaxy are considered to be foregrounds, which we remove to isolate the cosmological signal. We estimate the extragalactic contribution as $\mathrm{DM}_{\mathrm{EG}}=\mathrm{DM}_{\text {Measured }}-\mathrm{DM}_{\mathrm{MW}}$, ignoring the host contribution which scales as $(1+z)^{-1}$ for cosmological FRBs. The Milky Way contribution based on the NE2001 Galactic electron density model (Cordes \& Lazio 2002) is given by Petroff et al. (2015) for each FRB location.

Next, we estimate the intrinsic luminosity of the bursts at their rest-frame central frequencies $\nu_{0}=\bar{\nu}(1+z)$

$$
L_{\nu_{0}}^{\text {peak }}=\frac{4 \pi D_{L}^{2}\left(z_{0}\right) S_{\bar{\nu}}^{\text {peak }}}{\left(1+z_{0}\right)},
$$

where $\bar{\nu}$ is the mean observed frequency, $D_{L}\left(z_{0}\right)$ is the luminosity distance, assuming the source emission is isotropic. We also assume a flat spectral energy distribution since there is no spectral information available for most of the detected FRBs with the exception of the burst detected in the Arecibo Survey (Spitler et al. 2014) and the recently detected FRB150418. Finally, we choose four representative FRBs out of the observed ones selecting an FRB with the Maximal (excluding the "Lorimer" burst), Median and Minimal intrinsic luminosity densities and also considering the the "Lorimer" burst. The details $\left(z_{0}, \nu_{0}, L_{\nu_{0}}^{\text {peak }}\right)$ of these bursts are summarized in Table 1.

\subsection{Prospects of Observation with $S K A$}

We consider the four representative FRBs (Table 1) as four types of sources with different characteristic lumi- 
TABLE 1

Selected FRBs from the CAtalog of Petroff et Al. (2016) With the minimal, median, and maximal (excluding the LORIMER BURST) INTRINSIC PEAK DIFFERENTIAL LUMINOSITY AND THE LORIMER BURST.

\begin{tabular}{|c|c|c|c|c|c|c|}
\hline Type & Event & Telescope & $\mathrm{DM}_{\mathrm{EG}}\left[\mathrm{pc} \mathrm{cm} \mathrm{cm}^{-3}\right]$ & $z_{0}$ & $\nu_{0}[\mathrm{GHz}]$ & $L_{\nu_{0}}^{\text {peak }}\left[\mathrm{erg} \mathrm{s}^{-1} \mathrm{~Hz}^{-1} \mathrm{sr}^{-1}\right]$ \\
\hline Min & FRB010621 (Keane et al. 2011) & Parkes & 748 & 0.22 & 1.7 & $5.2 \times 10^{32}$ \\
\hline Median & FRB110523 (Masui et al. 2015) & GBT & 623 & 0.55 & 1.2 & $5.0 \times 10^{33}$ \\
\hline Max & FRB110220 (Thornton et al. 2013) & Parkes & 944 & 0.85 & 2.55 & $2.6 \times 10^{34}$ \\
\hline Lorimer & FRB010724 Lorimer et al. (2007) & Parkes & 375 & 0.32 & 1.86 & $>8.2 \times 10^{34}$ \\
\hline
\end{tabular}

nosities, i.e., with their intrinsic luminosity $L_{\nu_{0}}^{\text {peak }}$ fixed, and estimate the signal to noise with which each type can be probed with the SKA. For each burst we reverse the Eq. (7) and find the peak flux of each FRB varying its redshift $z_{F R B}$, or, equivalently, its observed frequency $\nu_{F R B}$. The expected scaling of the signal with $\nu_{F R B}$ is shown on the left panel of Figure 3 for each type of the FRBs together with the expected $10 \sigma$ sensitivity of SKA at each frequency shown for Phase 1 and 2 of the SKAMID in Band $1(0.35-0.95 \mathrm{GHz})$ and Band $2(0.95-1.76$ $\mathrm{GHz})$ and of SKA-LOW $(50-350 \mathrm{MHz})$. For both SKAMID, which is an array of dish antennas, and SKA-LOW, an array of low-frequency dipole antennas, the sensitivity is estimated as follows

$$
\sigma=\frac{\mathrm{SEFD}}{\sqrt{2 t_{\mathrm{int}} \Delta \nu_{\mathrm{BW}}}},
$$

where $t_{\text {int }}$ is the integration time, $\Delta \nu_{\mathrm{BW}}$ is the bandwidth and factor 2 of accounts for 2 polarization channels. $\mathrm{SEFD} \equiv 2 k_{B}\left(A_{e} / T_{s y s}\right)^{-1}$ measured in $\mathrm{Jy}$, is the system equivalent flux density which depends on the effective area, $A_{e}$, and the system temperature, $T_{s y s}$, which for Phase 1 of the SKA can be found in the SKA online documentation 2 for the entire SKA-LOW array and for a single dish of SKA-MID. To estimate the sensitivity of Phase 1 of SKA-MID we assume that the effective area of the array is 133 times the area of a single dish. Based on the SKA online documentation we assume that Phase 2 of SKA-MID will be 10 times better than Phase 1 in terms of SEFD, and SKA-LOW will be 4 times better. On the right panel of Figure 3 we show the signal to noise of each FRB as a function of redshift with horizontal lines marking the $10 \sigma, 5 \sigma$ and $1 \sigma$ detection thresholds.

According to our estimates and in agreement with previous studies (Macquart et al. 2015), SKA-MID will be a very powerful observatory for detecting all the FRBs except for the weakest ones, to which Band-2 of SKAMID should still be sensitive enough. On the other hand, Phase 1 of SKA-LOW is expected to be sensitive only to the strongest FRBs of the Lorimer type, while Phase 2 can also be sensitive to the entire brightest half of the FRB population. Increasing integration time can be beneficial in this case since the bursts are stretched in time by the factor of $\left(1+z_{F R B}\right)$ and the delay time grows for higher redshift sources observed at low frequencies. With different integration times for SKA-MID $\left(t_{\text {int }}=1\right.$ $\mathrm{ms})$ and SKA-LOW ( $\left.t_{\text {int }}=100 \mathrm{~ms}\right)$, which explains the jump in signal to noise, all FRBs brighter or identical to the Median are detectable with high signal to noise, $\mathrm{S} / \mathrm{N}>10$, out to $z \sim 11$ and out to $z \sim 14$ with

\footnotetext{
${ }^{2}$ http://www.skatelescope.org/
}

high signal to noise $\mathrm{S} / \mathrm{N}>5$ even with SKA Phase 1; while weaker FRBs will be detectable with Phase 2 out $z \sim 10.4$ with $\mathrm{S} / \mathrm{N}>5$.

With current constraints of the reionization history, FRBs at redshift $\sim 10$ are expected have a very large DM of several thousands (Figure 1). To allow for detection of so high DM with a telescope, one simply needs to assume that the maximal dispersion smearing within a frequency channel, $\Delta t_{\max }=8.3 \times 10^{-3} \mathrm{DM}_{\max } \Delta \nu / \nu_{\text {min }}^{3}$ seconds, is bigger than the sampling time, $t_{\text {samp. }}$. Here $\Delta \nu$ is the frequency channel width in $\mathrm{GHz}$, and $\nu_{\min }$ is the minimum (lowest) frequency in the observation band. Thus, the maximal DM that can be probed by a telescope is

$$
\mathrm{DM}_{\text {max }}=\frac{t_{\mathrm{samp}}}{8.3 \times 10^{-3} \mathrm{~s}} \frac{\nu_{\text {min }}^{3}}{\Delta \nu} .
$$

This argument should equally apply for both dishes (such as SKA-MID) and a low-frequency array (such as SKA-LOW). Most of the known FRB were detected with cutoff implied on the DM at $\mathrm{DM}_{\max } \sim 2000 \mathrm{pc}$ $\mathrm{cm}^{-3}$ (Masui et al. 2015; Thornton et al. 2013). However, higher DMs are being accessed with ongoing surveys. For example, Burke-Spolaor \& Bannister (2014) searched DM trials up to $3000 \mathrm{pc} \mathrm{cm}^{-3}$; while a recently developed pipeline allows the data searched over the DM from 0 to $5000 \mathrm{pc} \mathrm{cm} \mathrm{cm}^{-3}$ and over widths from 0.128 to $262 \mathrm{~ms}$ (Keane \& Petroff 2015). However, no FRBs with the DM above $1700 \mathrm{pc} \mathrm{cm}{ }^{-3}$ were detected so far. Future instruments, such as the SKA, will allow one to explore higher values of the DM. In particular, with the Phase 1 of the SKA the search out to dispersion measures of $3000 \mathrm{pc} \mathrm{cm}^{-3}$ is proposed allowing to probe FRBs out to $z \sim 3$, while with SKA Phase-2 it should be possible to search out to dispersion measures of $10,000 \mathrm{pc} \mathrm{cm}^{-3}$ and beyond (Keane et al. 2015) probing the FRB population during the EoR.

In the above discussion we assumed that there is no significant scattering in the intergalactic medium. With scattering, the observed flux is expected to decrease (Hassall et al. 2013). However, for all the observed FRBs the scattering by the IGM was estimated to be small (Thornton et al. 2013). Taking this into account together with the fact that the peak flux could have been missed by observation, the signal which we quoted above (and, thus, the signal-to-noise values) should be regarded as lower limits only.

\section{CONCLUSIONS}

In this Letter we explored the connection between the DM of high-redshift radio transients and the total CMB optical depth $\tau_{C M B}$ by deriving an analytic $\tau$-DM relation, which can be used to constrain $\tau_{C M B}$ provided radio 

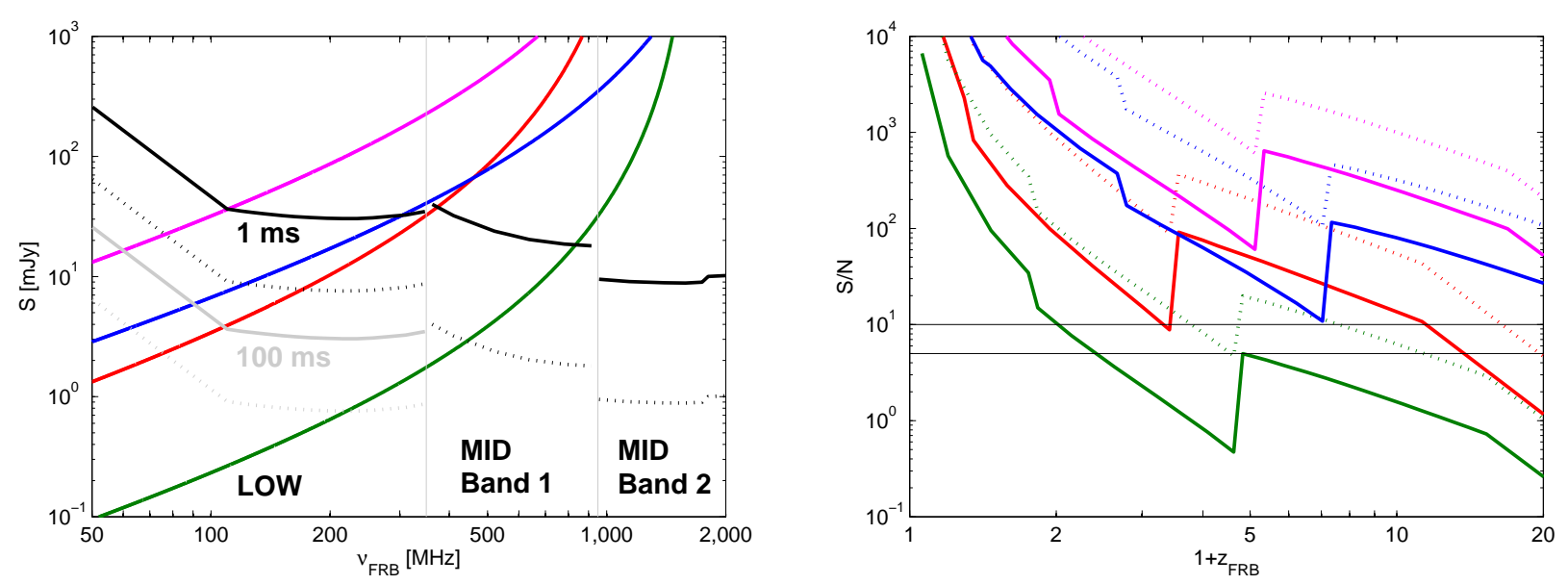

FIG. 3.- Left: Prospect of detection for the four selected FRBs listed in Table 1 placed at different cosmological distances. We show Minimal (green), Median (red), Maximal (blue) and Lorimer (magenta), together with the 5- $\sigma$ noise level of Phase 1 (solid) and Phase 2 (dotted) of SKA-MID with 1 ms integration time, and SKA-LOW with $1 \mathrm{~ms}$ (black) and $100 \mathrm{~ms}$ (grey) integration times. Right: Signal to noise ratio for each FRB as a function of its redshift. We show the results for Phase 1 (solid) and Phase 2 (dotted) of SKA for Minimal (green), Median (red), Maximal (blue) and Lorimer (magenta) FRBs. We set an integration time of $1 \mathrm{~ms}$ for SKA-MID and $100 \mathrm{~ms}$ for SKA-LOW. Horizontal lines mark 10 and 5 signal-to-noise thresholds.

transients exist during reionization. Assuming sources, such as FRBs, exist at high redshifts, we have shown that Phase 1 of the SKA or a similar radio telescope should be able to probe the brightest half of the population out to high redshifts, $z \sim 14$. In this case, it should be possible to test the full reionization history with Phase 1 of the SKA through DM of these sources. According to the latest estimates, FRBs are frequent events, with $\sim 6000$ of them occurring every day (Champion et al. 2015), and at least one of them was confirmed to be extragalactic (Keane et al. 2016). Therefore, there are good chances for detection of more FRBs at cosmological distances with future telescopes, such as the SKA, espe- cially when exploring new region of the parameter space, such as higher dispersion measures and lower frequencies. Moreover, depending on the luminosity function of the population, high redshift sources could be gravitationally lensed by the foreground large scale structure, and, thus, more easily observed (Wvithe et al. 2011; Fialkov \& Loeb 2015).

We thank Mark Reid and Jim Moran for useful discussions. This work was supported in part NSF grant AST-1312034 (for A.L.).

\section{REFERENCES}

Planck Collaboration; Ade, P. A. R., et al. 2015, arXiv:1502.01589 Becker, G. D., Bolton, J. S., Madau, P., et al. 2015, MNRAS, 447, 3402

Burke-Spolaor, S., \& Bannister, K. W., 2014, ApJ, 792, 19

Champion, D. J., Petroff, E., Kramer, M., Keith, M. J., Bailes, M., et al., 2015, arXiv:1511.07746

Cohen, A., Fialkov, \& A., Barkana, R., 2015, arXiv:1508.04138

Cordes, J. M. \& Lazio, T. J. W. 2002, arXiv:0207156

Dolag, K., Gaensler, B. M., Beck, A. M., \& Beck, M. C. 2015, MNRAS, 451, 4277

Falcke, H. \& Rezzolla, L. 2014, A\&A, 562, 137

Fialkov, A. \& Loeb, A., 2016, arXiv:1601.03058

Fialkov, A. \& Loeb, A., 2015, ApJ, 806, 256

Geng, J. J. \& Huang, Y. F. 2015, ApJ, 809, 24

George, E. M., Reichardt, C. L., Aird, K. A., Benson, B. A., Bleem, L. E., et al. 2015, ApJ, 799, 177

Ginzburg V.L., 1973, Nature, 246, 415

Hassall, T. E., Keane, E. F., \& Fender, R. P., 2013, MNRAS, 436, 317

Ioka, K., 2003, ApJ 598, L79

Inoue, S., 2004, MNRAS 348, 999

Keane, E. F., Kramer, M., Lyne, A. G., Stappers, B. W., McLaughlin, M. A., 2011, MNRAS, 415, 3065

Keane, E., Bhattacharyya, B., Kramer, M., Stappers, B., Keane, E. F., et al., 2015, "A Cosmic Census of Radio Pulsars with the SKA ", Proceedings of Advancing Astrophysics with the Square Kilometre Array (AASKA14). 9 -13 June, 2014. Giardini Naxos, Italy.

Keane, E. \& Petroff, E., 2015, MNRAS, 447, 2858

Keane, E. F., Johnston, S., Bhandari, S., Barr, E., Bhat, N. D. R., et al., 2016, Nature, 17140
Kulkarni, S. R., Ofek, E. O., Neill, J. D., Zheng, Z., \& Juric, M. 2014, ApJ, 797, 70

Katz, J. I., arXiv:1505.06220

Liu, A., Pritchard, J. R., Allison, R., Parsons, A. R., Seljak, U., Sherwin, B. D., 2015, arXiv:1509.08463

Loeb, A. \& Furlanetto, S. 2013, The First Galaxies in the Universe, Princeton University Press (Princeton)

Loeb, A., Shvartzvald, Y., Maoz, D., 2014, MNRAS, 439, 46

Lorimer, D. R., Bailes, M., McLaughlin, M. A., Narkevic, D. J., \& Crawford, F. 2007, Sci, 318, 777

Lyubarsky, Y. 2014, MNRAS, 442, L9

Macquart, J. P., Keane, E., Grainge, K., McQuinn, M., Fender, R., et al., 2015, Proceedings of Advancing Astrophysics with the Square Kilometre Array (AASKA14). 9 -13 June, 2014. Giardini Naxos, Italy.

Masui, K., Lin, H.-H., Sievers, J., Anderson, C. J., Chang, T.-C., et al., 2015, Nature, 528, 523

Mingarelli, C. M. F., Levin, J., Lazio, T. J. W., 2015, ApJ, 814, 20

Palmer, D. M., 1993, ApJ, 417, L25

Petroff, E., Bailes, M., Barr, E. D., Barsdell, B. R., Bhat, N. D. R. et al., 2015, MNRAS, 447, 246

Petroff, E., Barr, E. D., Jameson, A., Keane, E. F., Bailes, M., et al., 2016, arXiv:1601.03547

Ravi V., Shannon R. M., Jameson A., 2015, ApJ, 799, L5

Spitler L. G., Cordes, J. M., Hessels, J. W. T., Lorimer, D. R., McLaughlin, M. A. et al., 2014, ApJ, 790, 101

Totani, T. 2013, PASJ, 65, L12

Thornton, D., Stappers, B., Bailes, M., et al. 2013, Science, 341, 53

Wyithe, J. S. B., Yan, H., Windhorst, R. A., Mao, S., 2011, Nature, 469, 181 
Zahn, O., Reichardt, C. L., Shaw, L., Lidz, A., Aird, K. A., et al. $\quad$ Zhang, B. 2014, ApJ, 780, L21 2012, ApJ, 756, 65 